\title{
A microscopic theory of photonucleation: Density functional approach to the properties of a fluid of two-level atoms, a part of which is excited*
}

\author{
O.Derzhko' ${ }^{1}$, V.Myhal ${ }^{2}$ \\ ${ }^{1}$ Institute for Condensed Matter Physics of the National Academy of Sciences of Ukraine, \\ 1 Svientsitskii Str., 79011 Lviv, Ukraine \\ 2 The Ivan Franko National University of L'viv, Department for Theoretical Physics, \\ 12 Drahomanov Street, 79005 L'viv, Ukraine
}

Received August 26, 2005

We use the density functional method to examine the properties of the nonuniform (two-phase) fluid of twolevel atoms, a part of which is excited. From the analysis of the equation of state of a gas of two-level atoms, a part of which is excited, the following density functional of the grand thermodynamical potential emerges

$$
\Omega[\rho(\mathbf{r})]=\Omega_{\mathrm{CS}}[\rho(\mathbf{r})]-\frac{6 \sigma^{3} a\left(c_{1}, T\right)}{\pi} \int_{\left|\mathbf{r}_{1}-\mathbf{r}_{2}\right| \geqslant 2 \sigma} \mathrm{d} \mathbf{r}_{1} \mathrm{~d} \mathbf{r}_{2} \frac{\rho\left(\mathbf{r}_{1}\right) \rho\left(\mathbf{r}_{2}\right)}{\left|\mathbf{r}_{1}-\mathbf{r}_{2}\right|^{6}}
$$

with

$$
a\left(c_{1}, T\right)=\frac{1}{32} a^{2} v\left(E_{1}-E_{0}\right)\left(c_{0}-c_{1}+2 c_{0} c_{1} \frac{E_{1}-E_{0}}{k T}\right)
$$

(here $\Omega_{\mathrm{CS}}[\rho(\mathbf{r})]$ is the Carnahan-Starling term, $\sigma$ is the atom radius, $v=4 / 3 \pi \sigma^{3}, c_{1}$ is the concentration of excited atoms, $c_{0}+c_{1}=1, E_{1}-E_{0}$ is the excitation energy and $a$ is the dimensionless parameter which characterizes the atom). We use this expression to calculate the nucleation barrier for vapor-to-liquid phase transition in the presence of excited atoms.

Key words: photonucleation, nucleation barrier, density functional approach

PACS: $64.70 . F x, 82.65 . D p, 62.60 . N h, 64.60 . Q b$

The studies of equilibrium properties of a gas of identical atoms, a part of which is in an excited electronic state, have attracted attention for the last forty years [1-10]. Such atoms may appear due to the electromagnetic irradiation with the frequency, which corresponds to the excitation energy of the atom. Since the life-time of the excited state is essentially larger than the time required for establishing the equilibrium over translational degrees of freedom, the system should exhibit equilibrium properties at a given (nonequilibrium) concentration of excited atoms. Moreover, owing to new effective long-range interatomic interactions - the resonance dipole-dipole interactions - one may expect essential changes of various equilibrium characteristics due to the presence of excited atoms. The analysis performed within the framework of the cluster expansion method confirmed these expectations (see $[7,8]$ and references therein). On the other hand, there are only a few papers which may be related to experimental observations of theoretically investigated features of the gas with excited atoms. We should mention here the papers on the effect of irradiation on the condensation of iodine and anthracene vapor [11,12] and on the photonucleation [13-21], in particular, in vapors of mercury and cesium. The latter studies reported the quantitative results of resonance irradiation effect on the nucleation rate. The main conclusion of these studies is as follows: the resonance irradiation of the nucleation zone leads to a sharp increase of the nucleation rate. The experimental results, to our best knowledge, have not been explained so far. One may

*The paper submitted to the Proceedings of the conference "Statistical physics 2005: Modern problems and new applications" (August 28-30, 2005, Lviv, Ukraine). 
try to interpret these data basing on the theory of nucleation in a supersaturated vapor which contains excited atoms. Such atoms may appear as a result of resonance irradiation.

In what follows we present preliminary results about the nucleation phenomena in a fluid of two-level atoms, a part of which is excited (see also [22]). In our study we use the density functional approach developed by D.W. Oxtoby with coworkers [23]. This method permits to obtain the nucleation rate basing on the first principles. The nucleation rate $J$ is connected with the nucleation barrier $A, J=J_{0} \exp \left(-\frac{A}{k T}\right)$. The nucleation barrier can be calculated within the framework of the classical nucleation theory which relies on the capillarity approximation [23]

$$
\frac{A^{\mathrm{cl}}}{k T}=\frac{16 \pi}{3}\left(\frac{\gamma}{k T}\right)^{3} \frac{1}{\rho_{l}^{2} \ln ^{2} s} .
$$

Here $\gamma$ is the surface tension of the vapor-liquid interface, $\rho_{l}$ is the density of liquid, $s=p / p_{0}$ is the supersaturation, $p$ is the actual pressure of the supersaturated vapor and $p_{0}$ is the equilibrium pressure. To compute the nucleation rate according to equation (1) one has at first to construct the vapor-liquid phase diagram determining the equilibrium pressure $p_{0}$ and the liquid density $\rho_{l}$ at the temperature $T$ and then to compute the surface tension $\gamma$ at this temperature. This calculation can be done within the density functional approach considering the planar vapor-liquid interface. An alternative approach to the calculation of the nucleation barrier which does not use the key assumption in the classical nucleation theory - the capillarity approximation - was suggested by D.W. Oxtoby [23]. According to this scheme one has to consider a metastable vapor in a spherical vessel of the radius $\mathcal{R}$, assume the appearance of a spherical liquid droplet in the center of the vessel and analyse the density profile and the value of the grand thermodynamical potential of such a two-phase fluid with a spherical vapor-liquid interface. The value of the grand thermodynamical potential of such a metastable fluid $\Omega(T, \mu, V)$ permits to calculate the nucleation barrier via the equation

$$
A=\Omega(T, \mu, V)-\left(-p \frac{4}{3} \pi \mathcal{R}^{3}\right) .
$$

In our study we use both schemes in calculating the vapor-to-liquid nucleation barrier in a system of two-level atoms, a part of which is excited.

To perform the theoretical analysis of the vapor-to-liquid nucleation in the presence of the excited atoms we need an appropriate density functional of the grand thermodynamical potential. We assume the grand thermodynamical potential to be a functional of the density with the form

$$
\begin{aligned}
\Omega[\rho(\mathbf{r})]= & k T \int \mathrm{d} \mathbf{r}_{1} \rho\left(\mathbf{r}_{1}\right)\left(\ln \left(\Lambda^{3} \rho\left(\mathbf{r}_{1}\right)\right)+\frac{-1+6 v \rho\left(\mathbf{r}_{1}\right)-4 v^{2} \rho^{2}\left(\mathbf{r}_{1}\right)}{\left(1-v \rho\left(\mathbf{r}_{1}\right)\right)^{2}}\right) \\
& -\frac{6 \sigma^{3} a\left(c_{1}, T\right)}{\pi} \int_{\left|\mathbf{r}_{1}-\mathbf{r}_{2}\right| \geqslant 2 \sigma} \mathrm{d} \mathbf{r}_{1} \mathrm{~d} \mathbf{r}_{2} \frac{\rho\left(\mathbf{r}_{1}\right) \rho\left(\mathbf{r}_{2}\right)}{\left|\mathbf{r}_{1}-\mathbf{r}_{2}\right|^{6}}-\mu \int \mathrm{d} \mathbf{r}_{1} \rho\left(\mathbf{r}_{1}\right)
\end{aligned}
$$

where

$$
a\left(c_{1}, T\right)=\frac{a^{2}}{32} v\left(E_{1}-E_{0}\right)\left(1-2 c_{1}+2\left(1-c_{1}\right) c_{1} \frac{E_{1}-E_{0}}{k T}\right) .
$$

Here $\Lambda$ is thermal de Broglie wavelength of the atom, $v=4 / 3 \cdot \pi \sigma^{3}, \sigma$ is the radius of the atom, $c_{1}$ is the concentration of the excited atoms, $a=d^{2} \sigma^{-3}\left(E_{1}-E_{0}\right)^{-1}$ is the dimensionless parameter which characterizes the two-level atom (in what follows we set $a=1$ ), $E_{1}-E_{0}$ is the excitation energy, $d$ is the value of the transitional electrical dipole moment between the ground and excited states. The equilibrium density minimizes the grand thermodynamical potential $\Omega[\rho(\mathbf{r})]$, i.e. it is the solution of the following integral equation

$$
\begin{aligned}
k T \ln \left(\Lambda^{3} \rho\left(\mathbf{r}_{1}\right)\right) & +k T \frac{8 v \rho\left(\mathbf{r}_{1}\right)-9 v^{2} \rho^{2}\left(\mathbf{r}_{1}\right)+3 v^{3} \rho^{3}\left(\mathbf{r}_{1}\right)}{\left(1-v \rho\left(\mathbf{r}_{1}\right)\right)^{3}} \\
& -\frac{12 \sigma^{3} a\left(c_{1}, T\right)}{\pi} \int_{\left|\mathbf{r}_{1}-\mathbf{r}_{2}\right| \geqslant 2 \sigma} \mathrm{d} \mathbf{r}_{2} \frac{\rho\left(\mathbf{r}_{2}\right)}{\left|\mathbf{r}_{1}-\mathbf{r}_{2}\right|^{6}}-\mu=0 .
\end{aligned}
$$


Substituting the equilibrium density into equation (3) one obtains the value of the grand thermodynamical potential of the system $\Omega(T, \mu, V)$. The adopted density functional of the grand thermodynamical potential (3) is consistent with the virial state equation obtained earlier [1]. It takes into account the short-range interaction within the Carnahan-Starling local approximation (the first term in the r.h.s. of equation (3)) neglecting the difference of the atom radii in the ground and excited states. Moreover, it takes into account the long-range interactions, in particular, the resonance dipole-dipole interactions, within the mean-field approximation (the second term in the r.h.s. of equation (3)). Note, that the coefficient $a\left(c_{1}, T\right)(4)$ depends on temperature only when $c_{1}$ deviates from zero. More sophisticated density functionals are available but they have not been employed in the present study.

It is convenient to introduce the dimensionless units of energy, temperature, chemical potential, length, volume, density, pressure, surface tension etc renormalizing these quantities as follows: $E \rightarrow \frac{E}{E_{1}-E_{0}}, T \rightarrow \frac{k T}{E_{1}-E_{0}}, \mu \rightarrow \frac{\mu}{E_{1}-E_{0}}, r \rightarrow \frac{r}{\sigma}, V \rightarrow \frac{V}{v}, \rho \rightarrow v \rho, p \rightarrow \frac{p v}{E_{1}-E_{0}} \gamma \rightarrow \frac{\gamma \sigma^{2}}{E_{1}-E_{0}}$ etc, respectively. As a result equations $(3),(4),(5)$ become

$$
\begin{gathered}
\Omega[\rho(\mathbf{r})]=\frac{3 T}{4 \pi} \int \mathrm{d} \mathbf{r}_{1} \rho\left(\mathbf{r}_{1}\right)\left(\ln \left(\frac{\Lambda^{3}}{v} \rho\left(\mathbf{r}_{1}\right)\right)+\frac{-1+6 \rho\left(\mathbf{r}_{1}\right)-4 \rho^{2}\left(\mathbf{r}_{1}\right)}{\left(1-\rho\left(\mathbf{r}_{1}\right)\right)^{2}}\right) \\
-\frac{9 \alpha\left(c_{1}, T\right)}{2 \pi^{2}} \int_{\left|\mathbf{r}_{1}-\mathbf{r}_{2}\right| \geqslant 2} \mathrm{~d} \mathbf{r}_{1} \mathrm{~d} \mathbf{r}_{2} \frac{\rho\left(\mathbf{r}_{1}\right) \rho\left(\mathbf{r}_{2}\right)}{\left|\mathbf{r}_{1}-\mathbf{r}_{2}\right|^{6}}-\frac{3 \mu}{4 \pi} \int \mathrm{d} \mathbf{r}_{1} \rho\left(\mathbf{r}_{1}\right), \\
\alpha\left(c_{1}, T\right)=\frac{a^{2}}{32}\left(1-2 c_{1}+\frac{2\left(1-c_{1}\right) c_{1}}{T}\right), \\
T \ln \left(\frac{\Lambda^{3}}{v} \rho\left(\mathbf{r}_{1}\right)\right)+T \frac{8 \rho\left(\mathbf{r}_{1}\right)-9 \rho^{2}\left(\mathbf{r}_{1}\right)+3 \rho^{3}\left(\mathbf{r}_{1}\right)}{\left(1-\rho\left(\mathbf{r}_{1}\right)\right)^{3}}-\frac{12 \alpha\left(c_{1}, T\right)}{\pi} \int_{\left|\mathbf{r}_{1}-\mathbf{r}_{2}\right| \geqslant 2} \mathrm{~d} \mathbf{r}_{2} \frac{\rho\left(\mathbf{r}_{2}\right)}{\left|\mathbf{r}_{1}-\mathbf{r}_{2}\right|^{6}}-\mu=0 .
\end{gathered}
$$

Moreover, we assume for concreteness in equations (4) and (7) $a=1$. We also set without loss of generality $\Lambda^{3} / v=1$.

We start with the phase diagram of the system. For this purpose we assume the constancy of the density, $\rho(\mathbf{r})=\rho$, that immediately yields instead of equations (6) and (8) the following expressions

$$
\Omega(\rho)=T \rho V\left(\ln \rho+\frac{-1+6 \rho-4 \rho^{2}}{(1-\rho)^{2}}\right)-\alpha\left(c_{1}, T\right) \rho^{2} V-\mu \rho V
$$

and

$$
T \ln \rho+T \frac{8 \rho-9 \rho^{2}+3 \rho^{3}}{(1-\rho)^{3}}-2 \alpha\left(c_{1}, T\right) \rho-\mu=0 .
$$

Solving equation (10) with respect to $\rho$ and substituting this density into equation (9) one gets the value of the grand thermodynamical potential $\Omega(T, \mu, V)$. One can also eliminate, using equation (10), the chemical potential $\mu$ from equation (9) thus getting the equation of state

$$
-\frac{\Omega(T, \rho, V)}{T V}=\frac{p}{T}=\rho \frac{1+\rho+\rho^{2}-\rho^{3}}{(1-\rho)^{3}}-\frac{a\left(c_{1}, T\right)}{T} \rho^{2} .
$$

Equation (11) agrees with the virial state equation of a gas of two-level atoms, a part of which is excited, obtained earlier [1] (see also [7,8]). Equation (10) may have more than one solution which yield the same value of the grand thermodynamical potential $\Omega(T, \mu, V)$. Indeed, for a given temperature $T$ let us fix the value of the grand thermodynamical potential $-\Omega / V=p$ and solve equation (11) with respect to $\rho$. At high temperatures (above the critical temperature $T_{\mathrm{c}}$ ) one finds only one solution $\rho$ which corresponds to a certain value of the chemical potential $\mu$ in equation (10). 
At low temperatures (below the critical temperature $T_{\mathrm{c}}$ ) one finds several solutions $\rho$ with the corresponding values of chemical potential $\mu$ which follow from equation (10). Varying the value of the grand thermodynamical potential $-\Omega / V=p$ one finds such two densities $\rho_{\mathrm{v}}$ and $\rho_{l}>\rho_{\mathrm{v}}$ which yield the same value of $\mu$. The quantities $T, p=p_{0}, \rho_{\mathrm{v}}, \rho_{l}, \mu=\mu_{0}$ correspond to the points on the phase diagram where the two phases, liquid and vapor, coexist (see figure 1 ). In figure 1
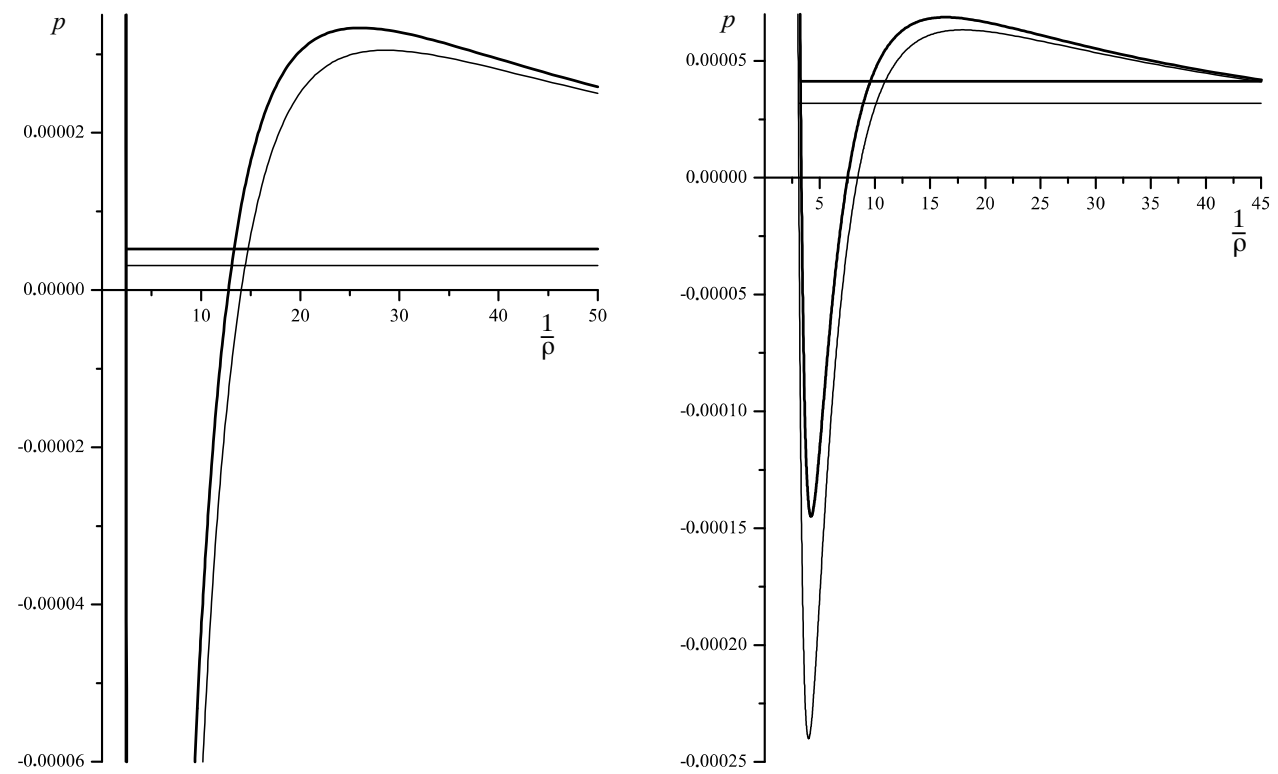

Figure 1. The isotherms $p$ vs $\rho^{-1}$ at $T=0.6 T_{\mathrm{c}}(0)$ (left panel) and $T=0.8 T_{\mathrm{c}}(0)$ (right panel). Bold curves correspond to $c_{1}=0$, thin curves correspond to $c_{1}=0.00006(=0.006 \%)$. The left (right) endpoint of the horizontal part of the isotherm (corresponding to $p_{0}$ ) gives the liquid density $\rho_{l}$ (the vapor density $\rho_{\mathrm{v}}$ ).

we display the isotherms which correspond to the temperatures $T=0.6 T_{\mathrm{c}}(0) \approx 0.00176866$ (left panel) and $T=0.8 T_{\mathrm{c}}(0) \approx 0.00235822$ (right panel) (here $T_{\mathrm{c}}(0)$ denotes the critical temperature $T_{\mathrm{c}}$ without excited atoms, i.e. when $c_{1}=0$ ) for two concentrations of excited atoms, $c_{1}=0$ (bold curves) and $c_{1}=0.00006(=0.006 \%)$ (thin curves). Considering at first the case $c_{1}=0$ at $T=0.8 T_{\mathrm{c}}(0)$ we find that the equilibrium values of the pressure, the chemical potential, the liquid density, and the vapor density are $p_{0} \approx 0.00004118, \mu_{0} \approx-0.00996108, \rho_{l} \approx 0.30719568$, and $\rho_{\mathrm{v}} \approx 0.02172324$, respectively. Assume further that in a system the concentration of excited atoms becomes $c_{1}=0.00006$. For such a fluid the equilibrium values of the pressure is $p_{0} \approx 0.00003179$ and the vapor with the pressure $\approx 0.00004118$ becomes metastable with the value of supersaturation parameter $s \approx 1.29559738$. Moreover, the equilibrium values of the chemical potential, the liquid density, and the vapor density of the fluid with $c_{1}=0.00006$ at $T=0.8 T_{\mathrm{c}}(0)$ are $\mu_{0} \approx-0.01049448$, $\rho_{l} \approx 0.32745048$, and $\rho_{\mathrm{v}} \approx 0.01596817$, respectively.

To calculate the vapor-to-liquid nucleation barrier according to equation (1) one has to find the surface tension $\gamma$. Analyzing the density profile for a planar vapor-liquid interface (for this purpose we consider a two-phase system in a cylinder of the radius $\mathcal{R}$ and the height $\mathcal{L}$ ) at $T=0.8 T_{\mathrm{c}}(0)$ and $c_{1}=0.00006$ and estimating $\Omega\left(T, \mu_{0}, V\right)$ we find according to the relation $\gamma \pi \mathcal{R}^{2}=\Omega\left(T, \mu_{0}, V\right)-$ $\left(-p_{0} \pi \mathcal{R}^{2} \mathcal{L}\right)$ the value of the surface tension $\gamma=0.00051195$. As a result one immediately gets the value of the vapor-to-liquid nucleation barrier $A / T \approx 68.3294$ (see figure 2 , dash-dotted curve 3 ). Obviously, since $A / T$ becomes now finite (and decreases as $c_{1}$ increases) the nucleation of liquid from vapor becomes now possible.

On the other hand, we can calculate the nucleation barrier based on the equation (2). First we estimate the Thompson radius $r^{\star}=2 \gamma\left(k T \rho_{l} \ln s\right)^{-1}$ at $T=0.8 T_{\mathrm{c}}(0)$ and $c_{1}=0.00006$. We obtain $r^{\star} / 2 \approx 6.614283$. 
We note that $r^{\star}$ is rather small which may be a reason to go beyond the classical nucleation theory since the capillarity approximation cannot be justified for such small droplets. Next we calculate the chemical potential for the supersaturated vapor with excited atoms according to equation (10) with $\rho \approx 0.02227178$ (this value of density follows from equation (11) for $c_{1}=0.00006, T=0.8 T_{\mathrm{c}}(0)$ and $p \approx$ $0.00004118), \mu=-0.01049448+0.00049852$, and analyse the density profile of a spherical droplet in the supersaturated vapor seeking for a "stable" value of the grand thermodynamical potential which plays the role of $\Omega(T, \mu, V)$ in equation (2) (for details see [23]). We find $A / T \approx 67.12$ that agrees with the value obtained within the framework of the classical nucleation theory. The described calculations have to be repeated for other values of concentration $c_{1}$. Moreover, we perform such calculations for several values of temperature. Some of our findings are collected in figure 2 .

The main conclusion which can be read off from figure 2 is as follows: the vapor after the appearance of excited atoms becomes metastable with $s>1$ and the nucleation barrier for vapor-to-liquid phase transition becomes essentially diminished. This outcome agrees with a naive expectation that the long-range resonance dipole-dipole interactions should act in favor of liquid formation in vapor. Although the present consideration permits to obtain the nucleation rate which can be measured experimentally much more work is required to compare theory and experiment. Firstly, we have to analyse in detail the results for nucleation rates $[15,16]$ obtained using the upward thermal diffusion cloud chamber setup [24]. Secondly, we should bare in mind that the fluids whose photonucleation has been studied have more complicated particle structure and interparticle interactions. The comparison with experiment can therefore be only qualitative at present, and in this respect our results are consistent with the data reported in $[15,16]$.

One of the authors (O.D.) is grateful to the DAAD for the support of his visit to PhilippsUniversität Marburg in the autumn of 1995. He wishes to thank Dr. Hermann Uchtmann for kind hospitality and many stimulating conversations.

\section{References}

1. Malnev V.N., Pekar S.I., Zh. Eksp. Teor. Fiz., 1966, 51, 1811 (in Russian).

2. Malnev V.N., Zh. Eksp. Teor. Fiz., 1969, 56, 1325 (in Russian).

3. Malnev V.N., Pekar S.I., Zh. Eksp. Teor. Fiz., 1970, 58, 1113 (in Russian).

4. Vdovin Yu.A., Zh. Eksp. Teor. Fiz., 1968, 54, 445 (in Russian).

5. Bortsaikin S.M., Kudrin L.P., Novikov V.M., Zh. Eksp. Teor. Fiz., 1971, 60, 83 (in Russian).

6. Makhviladze T.M., Saritchev M.E., Zh. Eksp. Teor. Fiz., 1976, 71, 1592 (in Russian).

7. Yukhnovskii I.R., Derzhko O.V., Levitskii R.R., Physica A, 1994, 203, 381.

8. Derzhko O., Levitskii R., Chernyavskii O., Condens. Matter Phys., 1995, 6, 35 (L'viv).

9. Chernyavskii O.I., Ukr. Fiz. Zh., 1996, 41, 811 (in Ukrainian).

10. Malnev V.N., Naryshkin R.A., Ukr. J. Phys., 2005, 50, 333.

11. Bezuglij B.A., Galashin E.A., Dudkin G.Ya., Pis'ma v Zh. Eksp. Teor. Fiz., 1975, 22, 76 (in Russian).

12. Galashin A. E., Galashin E.A., Dokl. Akad. Nauk SSSR, 1975, 225, 345 (in Russian).

13. Katz J.L., McLaughlin T., Wen F.C., J. Chem. Phys., 1981, 75, 1459. 
14. Chen C.-C., Katz J.L., J. Chem. Phys., 1988, 88, 5007.

15. Martens J.A.E. Dissertation, University of Marburg/Lahn, 1987 (in German).

16. Cha G.-S. Dissertation, University of Marburg/Lahn, 1992 (in German).

17. Baranovskii S.D., Dettmer R., Hensel F., Uchtmann H., J. Chem. Phys., 1995, 103, 7796.

18. Fisk J.A., Rudek M.M., Katz J.L., Beiersdorf D., Uchtmann H., Atmospheric Research, 1998, 46, 211.

19. Uchtmann H., Dettmer R., Baranovskii S.D., Hensel F., J. Chem. Phys., 1998, 108, 9775.

20. Uchtmann H., Kazitsyna S.Yu., Baranovskii S.D., Hensel F., Rudek M.M., J. Chem. Phys., 2000, 113, 4171.

21. Uchtmann H., Kazitsyna S.Yu., Hensel F., Zdimal V., Triska B., Smolik J., J. Phys. Chem. B, 2001, 105, 11754.

22. Derzhko O.V., Myhal V.M., J. Phys. Stud. (L'viv), 2005, 9, 156 (in Ukrainian).

23. Oxtoby D.W., J. Phys.: Condens. Matter, 1992, 4, 7627.

24. Katz J.L., J. Chem. Phys., 1970, 52, 4733.

\title{
Мікроскопічна теорія фотонуклеації: метод функціоналу густини для дослідження властивостей плину дворівневих атомів, частина з яких збуджена
}

\author{
О.Держко ${ }^{1}$, В.Мигаль ${ }^{2}$
}

1 Інститут фізики конденсованих систем НАН України, 79011 Львів, вул. Свєнціцького, 1

2 Львівський національний університет ім. І.Франка, факультет теоретичної фізики, 79005 Львів, вул. Драгоманова 12, Україна

Отримано 26 серпня 2005 р.

Ми використовуємо метод функціоналу густини для дослідження властивостей неоднорідного (двофазного) плину дворівневих атомів, частина з яких збуджена. На основі аналізу рівняння стану газу дворівневих атомів, частина з яких збуджена, виникає наступний функціонал густини великого термодинамічного потенціалу

$$
\Omega[\rho(\mathbf{r})]=\Omega_{\mathrm{CS}}[\rho(\mathbf{r})]-\frac{6 \sigma^{3} a\left(c_{1}, T\right)}{\pi} \int_{\left|\mathbf{r}_{1}-\mathbf{r}_{2}\right| \geqslant 2 \sigma} \mathrm{d} \mathbf{r}_{1} \mathrm{~d} \mathbf{r}_{2} \frac{\rho\left(\mathbf{r}_{1}\right) \rho\left(\mathbf{r}_{2}\right)}{\left|\mathbf{r}_{1}-\mathbf{r}_{2}\right|^{6}}
$$

3

$$
a\left(c_{1}, T\right)=\frac{1}{32} a^{2} v\left(E_{1}-E_{0}\right)\left(c_{0}-c_{1}+2 c_{0} c_{1} \frac{E_{1}-E_{0}}{k T}\right)
$$

(тут $\Omega_{\mathrm{CS}}[\rho(\mathbf{r})]$ - доданок Карнагана-Старлінга, $\sigma$ - радіус атома, $v=4 / 3 \pi \sigma^{3}, c_{1}-$ концентрація збуджених атомів, $c_{0}+c_{1}=1, E_{1}-E_{0}$ - енергія збудження і $a$ - безрозмірний параметр, який характеризує атом). Ми використовуємо цей вираз для обчислення нуклеаційного бар'єру для фазового переходу пари в рідину за наявності збуджених атомів.

Ключові слова: фотонуклеація, нуклеаційний бар'єр, метод функціоналу густини

PACS: $64.70 . F x, 82.65 . D p, 62.60 . \mathrm{Nh}, 64.60 . \mathrm{Qb}$ 